\title{
Do Large Probabilities Explain Better?
}

\author{
Michael Strevens \\ Philosophy of Science, 67:366-390, 2000.
}

\begin{abstract}
It is widely thought that the size of a probability makes no difference to the quality of a probabilistic explanation. I argue that explanatory practice in statistical physics, past and present, belies this claim. The claim has gained currency only because of an impoverished conception of the nature of probabilistic processes and an unwarranted assumption that all probabilistic explanations have a single form.
\end{abstract}

The last twenty-five years or so have seen a move away from the logical empiricists' inferential or expectability account of scientific explanation (Hempel and Oppenheim 1948) towards a causal account. At the same time, and for closely related reasons, there have been two trends in the treatment of the probabilistic explanation of events:

1. A move from accounts where only high probabilities explain to accounts where all probabilities can explain, and

2. A move from accounts where the quality of the explanation is proportional to the magnitude of the probability to accounts where all probabilities, regardless of size, explain equally well. 
This paper argues that (2) is a mistake. ${ }^{1}$ I will show (sections 2 and 3 ) that views on which the size of the probabilities involved in an explanation have no effect on the quality of the explanation-which I will call egalitarian accounts of probabilistic explanation-are unable to make sense of some of the most important probabilistic explanations in science, those of statistical physics. I then contend (section 4 ) that egalitarianism owes its appeal to an impoverished conception of the nature of probabilistic processes and to a mistaken belief that one cannot have (1) without (2). My conclusion is that, although low probabilities may have some explanatory power, the explanatory power of high probabilities is much greater.

\section{The Rise of Egalitarianism}

I will begin with a short history of the developments in the philosophy of probabilistic explanation that led from elitism to egalitarianism. The discussion is selective, and approaches to explanation-for example, the unification account - that have not made distinctive contributions concerning the underlying form of probabilistic explanation, are not mentioned. The concern with the explanation of events, as opposed to laws, reflects the focus of most of the relevant literature; however, as will become clear in sections 2.3, 2.4, and 3 , the debate about egalitarianism cannot be conducted without paying some attention to the explanation of laws.

1. To the best of my knowledge, there has been very little opposition to egalitarianism over the last twenty-five years. One exception is Mellor (1976). Mellor appeals not to explanatory practice in science, as I do, but to the felt explanatory force of high probabilities.

Notable among more recent writers are van Fraassen (1980, chap. 5) and Sober (1984, 142-7), who hold that (a) every explanation is with respect to a contrast case, and (b) the event being explained must be better probabilified by the explaining factors than is the contrast case. Although they are clearly not egalitarians, neither van Fraassen nor Sober make an independent case against egalitarianism.

Interestingly, all these writers accept, as I do, that events of low probability can, at least in some circumstances, be explained. 


\subsection{The Inductive-Statistical Account}

Throughout his career Carl Hempel advocated what might be called an expectability approach to scientific explanation (Hempel and Oppenheim 1948: Hempel 1965a). To explain an event, according to Hempel, is to use a set of facts, including at least one law of nature, to show that the event (called the explanandum) was to be expected. This is done by constructing a sound argument that has the law-including set of facts as its premises, and as its conclusion the proposition that the explanandum occurred.

Hempel held that, when deterministic laws are available, the argument for the explanandum must be deductive; that is, it must be the kind of argument that shows that the explanandum had to occur, and so could be expected with certainty (deductive-nomological, or DN, explanation). When only probabilistic laws are available, the argument must of necessity be inductive; that is, it must be an argument that shows that the explanandum had a high probability of occurring (inductive-statistical, or Is, explanation). Such an argument does not confer certainty on its conclusion, but as Hempel noted, a high probability is a sufficient basis for expecting an event to occur. ${ }^{2}$ What is more, only an event that can be shown to have a high probability can be reasonably expected to occur. It follows that, on an expectability account of probabilistic explanation such as Hempel's, only events with a high probability can be explained (Hempel 1965a, \$3.3). The Is account of probabilistic explanation is, in a very strong sense, an elitist account.

The Is account has a number of serious problems. One of the best known seems to arise from the account's elitism, and so was an early spur to egalitarian views. I refer to Michael Scriven's paresis counterexample (Scriven 1959.

2. At least, this is true provided that all relevant evidence has been taken into account. This proviso is reflected in the Is account by what Hempel calls the requirement of maximal specificity. Because the the requirement is somewhat complicated, yet has no bearing on the elitism of Is explanation, I will not discuss it here. The details may be found in Hempel (1965a \$3.4). 
480).$^{3}$ If syphilis in the latent stage goes untreated, there is a $25 \%$ chance that tertiary syphilis will develop, in which case there is a further small chance that the patient will acquire paresis (insanity brought on by the massive destruction of brain tissue). Untreated latent syphilis is, for this reason, generally held to explain the onset of paresis in any given patient. But the probability of a person's acquiring paresis given that they have untreated latent syphilis is very small. So a patient's having syphilis does not, on Hempel's account, qualify as an explanation of their succumbing to paresis-contrary to normal explanatory practice. This problem is not due to a merely technical flaw in the account; it is inherent in the expectability conception of explanation. For on any expectability account of explanation, to explain paresis, one would have to show that, given the patient's prior condition, paresis was to be expected. But it was not to be expected. Sometimes that which is unlikely can nevertheless be explained.

\subsection{The Statistical Relevance Account}

The successors to Hempel's account of probabilistic explanation accommodated the paresis counterexample by relaxing, one way or another, the high

probability requirement. The most influential treatment of the paresis case, and of probabilistic explanation in general, was Wesley Salmon's statistical relevance (SR) account (Salmon 1970).

Salmon summarizes his account as follows:

An explanation is an assembly of facts statistically relevant to the explanandum, regardless of the degree of probability that results (Salmon 1970, 11).

The sR account departs from Hempel's expectability account in several important ways:

3. The counterexample was originally directed at the DN account, before Hempel had given an account of probabilistic explanation. 
1. The form of an explanation is not an argument, but something with less inherent structure, an "assembly".

2. The relation between the explaining facts and the explanandum is one of statistical relevance. In other words, the explaining facts must affect the probability of the explanandum.

3. Neither the size of the probability conferred on the explanandum, nor the magnitude and direction of the probability change, make any difference to the force of the explanation. An event can be explained even by citing factors that lowered its probability. ${ }^{4}$

It is this third, egalitarian aspect of SR explanation that concerns me. Observe that, with the adoption of the statistical relevance account, egalitarianism comes to consist of three parts, the views that (a) the size of the probability conferred on the explanandum, (b) the size of the probability changes due to the statistically relevant factors, and (c) the direction of these changes, make no difference to the force of an explanation. The three claims are logically independent, but (a) leads naturally to (b) and (c): if the size of the probability ultimately conferred on the explanandum is irrelevant, why should one care how much or whether it is increased or decreased $:^{5} \mathrm{My}$ strategy will be, for the most part, to argue against (a).

Salmon, of course, is egalitarian in all three respects. To make the implications of Salmon's egalitarianism quite clear, consider the following possible statistical relevance relations between an event $E$ and a factor $A$ (I do not include any probability lowering relations because of Salmon's reservations described in note 4 ):

4. Salmon later clarified his position: a good SR explanation must state at least one factor that raised the probability of the explanandum (Salmon 1984, 46).

5. Indeed, given the unimportance of the size of this probability, why, from an explanatory point of view, care about probability changes-about statistical relevance-at all? The proponent of the SR account must answer that it is just a primitive fact about explanation that statistical relevance matters. The proponent of the causal account can, by contrast, appeal to the need to "understand the mechanism"; see section 1.3 
1. A raised the probability of $E$ just a fraction, from zero to little more than zero.

2. A raised the probability of $E$ from near zero to near one.

3. A raised the probability of $E$ from near one to slightly nearer one.

According to the SR account, any of these three relevance relations would provide the basis for an equally good explanation of $E$. It does not matter that in (2) A makes a vast difference and in (3) hardly any difference at all; provided that whatever relevance relations exist are reported accurately and fully, all sR explanations are equal. Salmon admits to a "feeling of queasiness" about this (Salmon 1970, 12), but he stands firm.

\subsection{The Causal Account}

The essence of the causal account of explanation is the idea that understanding an event is a matter of understanding the mechanism that causally produced it. What is it to understand a mechanism? According to the version of the causal account that I will consider here, understanding a mechanism is a matter of knowing all the physical facts about the mechanism and all the causal laws that govern the behavior of the mechanism. Put these facts and laws together, and you have what I will call a pure causal explanation of the events caused by the mechanism. ${ }^{6}$

The canonical pure causal view of explanation is, I think, Peter Railton's account (Railton 1978, 1981). Paul Humphreys (1981, 1989) presents a very similar account. ${ }^{7}$ Salmon, too, has now endorsed what I take to be a pure

6. An "impure" causal account is one that adds some additional criterion for explanatory relevance to the pure causal account, such as a requirement that the explanandum counterfactually depend on the explaining facts.

7. Both Railton and Humphreys restrict the phrase "causal explanation" to explanations involving deterministic processes. Thus they would not refer to their accounts, which of course take pains to accommodate probabilistic processes, as "pure causal" accounts. This is 
causal account (Salmon 1984). An early paper in the development of the pure causal account is Jeffrey (1969). Jeffrey, like all causal theorists, holds that to explain an outcome is to convey an understanding of the mechanism that produced the outcome (although he has in mind perhaps a less realist conception of cause than later proponents of the causal account). Not coincidentally, the same paper makes one of the earliest and most forceful cases for egalitarianism in probabilistic explanation.

Why are proponents of the pure causal account egalitarians? Whereas egalitarianism seems optional on the SR account, it is very difficult to avoid on the pure causal account. Egalitarianism follows from two views very closely associated with the pure causal view, the one concerning what makes a pure causal explanation good, the second concerning the way in which probabilities are to be integrated into a pure causal explanation:

1. What makes a pure causal explanation a good explanation is the accuracy and completeness of its description of the mechanism that produced the explanandum. (This aspect of the pure causal view is presented most clearly in Railton (1981), but Humphreys and Salmon seem to be broadly in agreement.) That the mechanism is of one kind rather than another makes no difference to the power of the explanation, provided that it is accurately and completely described.

2. Probabilistic processes, hence probabilities, are to be considered as a facet of the mechanism that produces the explanandum. Part of describing the mechanism is describing any probabilities that play a role in the mechanism ${ }^{8}$

my own term; it should be understood to apply not only to those aspects of Railton's and Humphreys' accounts that they themselves would classify as causal, but also to those that they would classify as probabilistic.

8. Humphreys, unlike most pure causal theorists, denies this. For him, probabilities are not in themselves explanatorily relevant at all (see section 4 ), thus their values have no place in a probabilistic explanation. This premise leads immediately, of course, to egalitarianism. 
It follows from these two assumptions that a probabilistic explanation of an event is good to the degree that it provides an accurate and complete description of the probabilities involved (as well as describing, of course, the other non-probabilistic facets of the mechanism). Whether the probabilities are low or high, lowered or raised, does not matter, provided that these features, and all others, are fully and correctly documented. The pure causal account of explanation, and the egalitarianism that goes along with it, are now widely accepted.

\section{Probabilistic Explanation in Statistical Mechanics}

Egalitarian accounts of explanation hold that events with low probabilities are just as well explained as events with high probabilities. According to the pure causal view, this is because explanation is simply a matter of describing causal mechanisms accurately and completely.

Perhaps the best developed, most deeply probabilistic science we have is statistical mechanics. In what follows, I show that in characteristic statistical mechanical explanations, the probability of the explanandum must be high.

\subsection{A Gas in a Box}

Consider the following very simple experiment. I have a box with a partition in the middle. On one side of the partition is a certain amount of some gas. On the other side is nothing, that is, a vacuum. Without opening the box, I retract the partition. The gas rushes to fill the other half of the box. After a moment, it is evenly distributed throughout the entire box.

Statistical mechanics (sm) explains this event as follows. ${ }^{9}$ The gas is made up of many particles, each careening around the container more or less at

9. The explanation I give is the sort provided by kinetic theory; it is the kind of explanation that is found in introductory textbooks. Kinetic theory is not confined to textbooks, however; it is important for understanding the behavior of systems not at equilibrium 
random. There is the same probability of finding a given particle anywhere in the space available. Once the partition is retracted, the whole box is available space. Therefore at any time shortly after retraction, the probability of finding a given particle anywhere in the box is equal. Put together the probabilities for each individual particle, and there is a very high probability of finding the particles of the gas approximately evenly distributed throughout the box. (This is true for the same reason that, if there is an equal probability of obtaining heads or tails on a coin toss, there is a very high probability that tossing many coins will produce approximately even numbers of heads and tails.) It is the fact that there is such a high probability of the gas distributing itself evenly, says SM, that explains why the gas is distributed evenly. This seems to be a clear case of elitist explanation.

\subsection{Biting the Bullet}

I will consider six egalitarian responses to this counterexample. The egalitarian's first response is simply to stick to their egalitarianism: SM may explain high probability events, but it explains low probability events just as well. An example will be helpful. Consider the remote possibility that, after the partition is removed, the gas remains in the half of the box where it starts out (an event so unlikely one would not expect it to occur even once in the lifetime of the universe). The egalitarian's claim is that SM provides just as good an explanation of this event as it does of the event of the gas spreading throughout the box.

One way to evaluate this claim is to recall the reason that statistical

(often under the guise of some other name such as "transport theory"). There is a more formal statistical mechanical explanation for equilibrium phenomena, due originally to Gibbs, which is different from, but not inconsistent with, the kinetic theory explanation. The Gibbs explanation is, even more than the kinetic theory explanation, clearly a variety of high probability explanation. Indeed, it cites nothing but the high probability of the gas coming to occupy the entire box. For a discussion of the foundational question of the relation between the kinetic theory and the Gibbs explanations, see Sklar (1993). 
mechanics was accepted, in the late nineteenth century, as a worthwhile theory. At the time, sm did not make novel predictions. ${ }^{10}$ but rather gave new explanations of certain kinds of events already known to occur, such as the dispersal of a gas or the cooling of a hot iron immersed in cold water. It is generally agreed that SM was accepted in large part because of the power of these new explanations.

What, then, was explanatorily new in SM? An SM explanation of, say, the cooling of the iron, has two parts:

The mechanical part: This consists in the claim that the heat of the iron is a kind of motion of the particles that make up the iron, and that the cooling occurs because the iron particles transfer some of their motion to the water particles, coming to have less motion in the process.

The statistical part: This consists in the demonstration that the process described in the mechanical part of the explanation has a very high probability of occurring.

Now, what was novel in statistical mechanics, when it was introduced in the latter half of the nineteenth century by Maxwell and Boltzmann, was not the mechanical part of its explanations. The idea that substances were made of particles and that heat was a kind of motion of these particles-call it the molecular theory of heat-was popular in the seventeenth century. But by the early nineteenth century, it had been eclipsed by the caloric theory, the idea that heat is a kind of fluid substance, largely because the caloric theory seemed to explain thermodynamic phenomena, such as the cooling of the iron, far better than the molecular theory (Brush 1983, 42). What was novel in statistical mechanics was the introduction of the statistical part of the explanation, so as to show that the kinds of processes posited by the molecular theory were highly likely to give rise to the observed behavior

10. One interesting exception is Maxwell's prediction that the coefficient of viscosity for a gas is independent of its density. 
of gases and heat. Thus what gave the old molecular theory a degree of explanatory power that matched, and eventually exceeded, that of the caloric theory, was probability - the high probability that sm ascribed to the behavior to be explained. ${ }^{11}$

If egalitarianism is correct, however, showing that a process that generates some event might possibly occur explains the event just as well as showing that the process has a high probability of occurring. It follows that the statistical part of an sm explanation adds nothing to the explanation, and so that, after Maxwell's and Boltzmann's work, sM explained the phenomena no better than did the seventeenth century molecular theory. Thus, egalitarianism completely fails to make sense of this aspect of the history of physics.

11. I have simplified this story in three ways. First, I have not mentioned the other important reason for the success of the caloric theory, the belief that light and heat are the same sort of thing, coupled with the idea that light is a kind of substance. However, this belief was abandoned only after Maxwell developed his theory of electromagnetism in the 1860 s, by which time SM was - thanks to the introduction of probability into the molecular theory-already gaining ground (Brush 1983 44).

Second, statistical mechanics did not replace the caloric theory directly. Rather, it replaced the theory that had, in the 1830s, replaced the caloric theory: the wave theory of heat. The short-lived success of the wave theory was due to continued adherence to the idea that heat and light are the same kind of stuff, together with the ascendence of the theory that light is a wave phenomenon (Brush 1983, 43-44).

Third, it is of course an exaggeration to say that the development of statistical mechanics in no way enriched the seventeenth century understanding of the mechanical processes underlying the thermodynamic and gas laws. Nevertheless, the mechanical understanding was largely in place, thanks in large part to the contributions of Clausius, by the time Maxwell and Boltzmann made the theory fully statistical. Among Clausius's achievements were: the inclusion of rotational energy as a component of heat, the explanation of diffusion as a consequence of molecular collisions, non-probabilistic molecular accounts of states of matter other than gases, and non-probabilistic molecular accounts of phase transitions. This contribution to the mechanical part of SM far surpassed any made by Maxwell or Boltzmann. Thus, were the explanatory power of sM concentrated in the mechanical part, Clausius would be regarded as the undisputed genius of SM, rather than following a distant third to Maxwell and Boltzmann.

It is also worth noting that mechanical understanding took a great leap forward after statistical mechanics was firmly established, due to the development of quantum mechanics in the 1920s, yet that this great leap was not seen as bringing about a fundamental revision of the explanatory structure of statistical mechanics-except insofar as it affected the values of statistical mechanics' probabilities. 
The egalitarian might counter that Maxwell and Boltzmann's work was of value wholly because it made the molecular theory better confirmed than it had previously been, by showing that the probability ascribed to the phenomena by the molecular theory-the likelihood, for Bayesian and other purposes - was higher than had been previously believed. This is a radical proposition: it amounts to the claim that Maxwell and Boltzmann enhanced sM's explanatory power not one degree. The ramifications of such a view are discussed at length at the end of section 3. For now, I remind the reader that the nineteenth century inference, by way of statistical mechanics, to the existence of molecules, is widely considered the paradigm inference to the best explanation.

\subsection{Statistical Mechanics Explains Only Frequencies}

A possible response to this line of reasoning constitutes the second egalitarian reply to my argument. It might be maintained that the achievement of statistical mechanics was not to explain particular events but to explain laws, in particular, the gas laws and the laws of thermodynamics. In what follows, I will focus on the thermodynamic laws.

Consider a typical qualitative statement of the second law of thermodynamics: heat never flows from a cold object to a hot object (without some equivalent compensation, that is, without at least as much heat flowing from a hot to a cold object somewhere else in the system, as in a refrigerator). It was this sort of statement that sm was supposed to have explained. But what is the relationship between SM and the statement that heat never flows from cold to hot? Statistical mechanics does not actually entail this statement. Rather, it entails that the statement has a very high probability of being true.

Here the egalitarian can take one of two paths. The first option is to construe SM's explananda as non-probabilistic generalizations about the frequencies of thermodynamic events. In the case of the second law, this would mean arguing that sM owed its success to its ability to explain the 
non-probabilistic fact that heat never flows from cold to hot. The second option is to construe sm's explananda as probabilistic laws. In the case of the second law, this would mean arguing that SM owed its success entirely to its ability to explain why heat has a very low probability of flowing from cold to hot. The latter claim is discussed as a separate reply below (section 2.4); in what follows I discuss the former claim.

Statistical mechanics explains the non-probabilistic fact that heat never flows from hot to cold in exactly the same way that it explains the particular event of, say, a hot iron cooling in cold water. The explanation has two parts: a mechanical part, where the process of cooling is characterized at the molecular level, and a statistical part, where it is shown that such processes have so high a probability that they are utterly unlikely to have a single observed exception in the life of the human race. As I have explained above, the distinctive contribution of SM is the statistical part of the explanation. Thus sm's great explanatory achievement was attained by showing that the regularities to be explained had a very high probability of obtaining. The egalitarian gains nothing, then, by moving from the explanation of events to the explanation of laws, if the laws are construed as non-probabilistic facts about frequencies of events.

The same kind of point might be made independently of the historical argument. Say that statistical mechanics offers an empty explanation of the second law of thermodynamics if it would explain any other possible law about the flow of heat equally well. Then, according to the egalitarian, sm's explanation of the second law is empty. For sm only distinguishes between the following two generalizations:

1. Heat never flows from a cold object to a hot object.

2. Heat never flows from a hot object to a cold object.

by assigning a high probability to the first and a low probability to the second. Since the magnitude of the probabilities is, according to the egalitarian, 
explanatorily irrelevant, sm explains both generalizations equally well. More precisely, though more longwindedly, were the second generalization to be true, sM would, according to the egalitarian, explain this fact just as well as SM actually explains the truth of the first generalization. I say that the explanation is empty because it conveys no understanding of why heat flows one way but not the other, which is just to say that it conveys no understanding of why the second law of thermodynamics is true. ${ }^{12}$

It is worth noting, perhaps, that this is an instance of a more general truth about egalitarianism. If the pertinent explanatory fact about some type of event $E$ is simply that, in circumstances $C$, it has a very high probability of occurring, then not only is the non-occurrence of an $E$ event just as well explained as the occurrence of an $E$ event, but over a longer period, $E$ events' occurring with one frequency is equally well explained as their occurring with any other frequency. Thus, according the egalitarian, a probabilistic explanation can never explain why an event occurs with one frequency rather than another.

\subsection{Statistical Mechanics Explains Only Probabilities}

The possibility arose above that statistical mechanics explains not events themselves, nor the frequencies of events, but the fact that certain probabilities attach to events (equivalently, that SM explains certain probabilistic laws). The third egalitarian reply is that probabilities are indeed all that SM explains, and that such explanations are sufficient to account for the explanatory appeal of sM in the nineteenth century. I will call the view that probabilistic explanations explain only probabilities explanatory probabil-

12. The idea that an event is not really understood if the theory that explains it would also have explained its non-occurrence equally well has attracted some opposition. (See Salmon 1990, 178-179; note, however, that the principle rejected by Salmon is a stronger one that omits the "equally well".) I think that it is much harder to oppose the same idea when it is applied, as here, not to single events, but to the explanation of generalizations. 
ism. $\cdot^{13}$ My objection to explanatory probabilism does not dispute that SM explains probabilities - this, all parties are agreed, it does, and indeed, all its explanations go by way of statistical mechanical probabilities. What I object to is the assertion that SM explains nothing but probabilities.

To take a particular example: according to explanatory probabilism, SM does not explain why a released gas fills its box evenly, nor why all such gases do so, but only why the gas has a high probability of filling the box evenly. Similarly, according to explanatory probabilism, sm does not explain why a released gas does not remain in its own half of the box, nor why no such gases ever do so, but only why the gas has a low probability of staying put.

How does explanatory probabilism make sense of the late nineteenth century discovery of statistical mechanics? In the early nineteenth century there was a certain set of explanatory demands: Why do gases always expand into the space available? Why do hot irons cool in water? With sm came the following achievement: it was explained why there was a very high probability of gases expanding, irons cooling, and so on. So far explanatory probabilism tells the usual story. But then comes the radical move. On the conventional story, by explaining why, say, there is a very high probability of hot irons cooling, sm thereby explains why hot irons cool. On the explanatory probabilist story, the high probabilities explained by sM do not explain why hot irons cool. The cooling of hot irons is left unexplained-indeed, if explanatory probabilism is correct, it is to this day inexplicable, except by a deterministic explanation that appeals to the exact initial conditions of every particle in the iron, the water, and the surrounding environment. As a kind of consolation prize for this explanatory shortfall, a new thing, a probability, is explained instead. But this probability has no explanatory power itself.

The conventional story and the explanatory probabilist story, differ, then,

13. For a clear statement of explanatory probabilism, see Papineau (1985. 71). There is perhaps a hint of explanatory probabilism to be found in Railton and especially in Humphreys, although both writers officially maintain the view that the event, not the probability, is the explanandum. 
on the significance of the probabilities that sm introduces in the course of its explanations. On the conventional story, these new theoretical entities are introduced as an explanatory means to an end, that end being the satisfaction of the original explanatory demand. On the explanatory probabilist story, the new theoretical entities do not satisfy the original explanatory demandindeed they cannot, being intrinsically non-explanatory entities-but must rather be regarded as an explanatory end in themselves. Therefore, they must be regarded as satisfying an explanatory demand that had not previously been made.

Here, I think, the explanatory probabilist has already lost the historical argument, as SM was clearly regarded as satisfying the earlier demand. Just about everyone, then and now, thinks that sm explains why hot irons cool. But explanatory probabilism might nevertheless be defended on the grounds that SM, although it did not satisfy the old demand, managed somehow to create a new explanatory demand.

What would be the source of the new explanatory demand? The answer, presumably, is that sm persuaded the world that certain probabilities existed. An explanation of the probabilities was then required. There is much reason to doubt this story, however. The truth seems to be that the probabilities of SM were, and are, tolerated only because of their explanatory and predictive power. In this respect, the probabilities are like any theoretical entity: they are posits that are made as means to explanatory and predictive ends. The suggestion that their existence is somehow independently established, and that it draws explanatory attention away from the original phenomena and towards the explanation of the theoretical entities themselves, gets the story entirely the wrong way around. On top of this, the probabilities of sm have ontological difficulties that ordinary theoretical entities do not: it is not even clear how probabilities could exist in deterministic systems like those of classical physics. Much of the debate about sm at the turn the last century revolved around the contention that SM, despite its apparent ability to satisfy 
the pre-existing explanatory demands, was an illegitimate theory precisely because it posited probabilities where there were clearly none.

In my view, explanatory probabilism makes sm into a kind of explanatory protection racket. A protection racket, rather than satisfying a previous demand for security, deliberately creates an insecure situation for which it then offers alleviation. According to explanatory probabilism, rather than satisfying any previous explanatory demand, sM created a new explanatory demand by introducing a set of entities, the statistical mechanical probabilities, that themselves explain nothing else. Kindly, sm then offers its services as an explainer of these entities-and only of these entities. Like organized crime, sm cures no maladies it does not itself create. As a consequence, were sM to be discarded, there would be no net loss in science's explanatory power. This is a reductio of explanatory probabilism. ${ }^{14}$

\subsection{Explanation in Statistical Mechanics Is Not Probabilistic}

A fourth way out for the egalitarian is to claim that statistical mechanics does not offer probabilistic explanations at all, because the "probabilities" that appear in such explanations are not real. The reason usually given for thinking that the probabilities are not real is that they, unlike the probabilities of quantum mechanics, are not irreducible. This line of argument depends, then, on two assumptions: that the probabilities of classical mechanics are not derived somehow from quantum probabilities, and that a probability that is not fundamental is in some sense not real. The first assumption may be unwarranted by the physics (Albert 1994), and the second is, I think, highly tendentious. But rather than attacking the assumptions directly, I will attack their consequences. If the assumptions were true then, once again, the explanatory power, and hence the appeal, of sm becomes mysteriousits explanations explicitly hinge, after all, on the supposedly non-existent

14. For a different kind of argument against explanatory probabilism, see Salmon (1988). 
probabilities. The objector owes us some alternative story about explanation in sm. Peter Railton (1981) has offered such a story; because of the length of the discussion, it is treated separately in section 3 . (Also considered in section 3 is the possibility that SM in reality has no explanatory power, therefore that there is nothing for the egalitarian to account for.) I postpone discussion of this particular egalitarian reply, then, until that section.

\subsection{Explanation in Statistical Mechanics Is Quasi-Deterministic}

A brief comment made by Richard Jeffrey (1969, 20-21) suggests a fifth egalitarian reply. Jeffrey writes that the explanations of statistical mechanics are "really beautiful" in the sense that the probability of the explanandum is "so close to 1 as to 'make no odds' in any gamble or other decision problem" (p. 20). Jeffrey is writing (in the passage cited at least) in the context of Hempel's expectability account of explanation, hence the reference to decision theory, but an egalitarian might hope that the conclusion survives the context, and that an exception can be made to the egalitarian rule for probabilities that are very close to 1 . This exception would justify treating events with very high probabilities as better explained than events with very low probabilities, as statistical mechanics seems to do.

If this strategy is to succeed, two claims must be established: first, that the explanation of events with very high probabilities can be treated as though it were a kind of deterministic explanation, and second, that this provides a basis for explanatory discrimination in favor of such events, or more to the point, for explanatory discrimination against the non-occurrence of such events. I will concede the first claim, purely for the sake of the argument, and focus on the second. ${ }^{15}$

Assume, then, that sm's explanation of events such as the gas's dispersal can be regarded as though it were deterministic, due to the very high

15. For the (I think, compelling) case against the first claim see Railton (1978, 212-3). 
probabilities involved. (This view should not be confused with Railton's deterministic account of sm explanation, discussed in section 3.) What the egalitarian must do, in order to avoid the objection I posed above, is to show that this somehow makes the explanation of a gas's dispersal better than the explanation of its staying put in its own half of the box. The task for the egalitarian, then, is to show that the SM explanation for the gas's staying put is less good than a deterministic explanation.

The egalitarian cannot perform this task by arguing that the gas's staying put is physically impossible, as it is quite possible, though highly improbable. This constitutes an important disanalogy with deterministic explanation, where the non-occurrence of the explanandum really is, given the actual conditions, impossible. To what else can the egalitarian appeal? Only to the causal account of explanation that motivates modern egalitarianism in the first place. But, according to the causal account, explanation is entirely a matter of understanding the mechanism by which the explanandum is caused. In the highly improbable case where a gas remains in its own half of the box, however, we do understand the mechanism, or at least, we understand it just as well as in the probable case. Thus the two explanations are equally good. More generally, on the causal account of explanation, probabilistic explanations in which the probabilities are fully and accurately described are just as good as any deterministic explanation, regardless of the probability of the explanandum.

\subsection{Moderate Egalitarianism}

The sixth reply involves a strategic weakening of the egalitarian position. The weakening, which assumes an SR form for probabilistic explanation, is as follows: it is conceded that an event is not explained unless at least one statistically relevant factor is cited that increased the probability of the explanandum. (This is a concession that Salmon and Humphreys have already made; see note 4.) Egalitarianism is thus compromised because the direction 
of the probability change is admitted to make an explanatory difference. I call this view moderate egalitarianism. It retains most of the features of egalitarianism; in particular, it holds that the magnitude of the probability change has no effect on explanatory power.

Why is it worth making this compromise? There is reason for hoping that moderate egalitarianism will handle the case of the gas in the box, provided that it is allowed that sm's explanatory work is all done by statistical relevance relations (a concession I will make for the sake of the argument). The argument is as follows: retracting the partition in the box raises the probability that the gas becomes evenly distributed, but lowers the probability that the gas stays put. An event that has its probability raised is, on the moderate egalitarian view, explained, whereas an event that does not have its probability raised is not explained. Thus in the case of the gas, the moderate egalitarian view is in accordance with the physicists' view that SM explains the gas's moving throughout the box but not its staying put.

One does not have to go far, however, to find a case where moderate egalitarianism fails. Consider: retracting the partition increases the probabilityfrom zero to very, very slightly above zero- that the gas will come to occupy all and only the other side of the box. Thus according to moderate egalitarianism, were the gas ever to occupy just the other side of the box, that event would be just as well explained by SM as the event of an even distribution. But of course this conclusion is unacceptable, for all the reasons given above. Moderate egalitarianism falters for the same reason as extreme egalitarianism: it fails to take into account the size of the increased probabilities.

Or consider the following more complicated scenario: suppose that the experiment takes place within the earth's gravitational field, and that as the partition is retracted, the box is swiveled so that the part of the box containing the gas is closer to the earth. This swiveling will increase the probability of any gas molecule's being found in the lower half of the box, even as the removal of the partition increases the probability of a molecule being found 
in the upper half. Thus swiveling increases the probability of the entire gas remaining in the lower half and thereby decreases the probability that the gas will become evenly spread throughout the box, while removing the partition decreases the probability of the gas remaining in the lower half and increases the probability of an even spread. In short, each event is probabilified by one factor and simultaneously de-probabilified by the other. ${ }^{16}$ If only the direction of probabilification were important, both events would be equally well explained. But they are not. What also matters, of course, is that one event was probabilified by a far greater amount than it was de-probabilified, the other de-probabilified by a far greater amount than it was probabilified. In the swiveling experiment, an even distribution ${ }^{17}$ is explained and staying put is not, because the probability of the even distribution is made very high while that of staying put remains very low.

\section{Railton's Non-Probabilistic Treatment of Statistical Mechanics}

In section 2.5, I raised the possibility that explanation in statistical mechanics is not, after all, probabilistic, and so cannot be used as evidence for or against theories of probabilistic explanation. Discussion of this possibility requires an appeal to some alternative view of sM explanation, and was, for reasons of length, deferred to the current section. In what follows I present, on behalf of the egalitarian, an alternative view of explanation in classical statistical

16. It is perhaps worth rehearsing the standard formula for positive statistical relevance. A factor $A$ is positively relevant to $E$ given other conditions $B$ just in case $P(E \mid A B)>P(E \mid B)$. (I ignore the matter of screening off, which is not relevant here.) This formula is satisfied when $A$ represents the swiveling, $B$ the removal of the partition, and $E$ the gas's staying in the lower half of the box. Some authors require that the formula be true for a variety of $B$ s (Humphreys calls this the invariance requirement). This condition is satisfied in my example: gravity always increases the probability of a particle being found lower in a box.

17. More accurately, it is a near-even distribution that is explained, reflecting the (very small) influence of the gravitational field on the probability distribution of the position of each particle. 
mechanics, due to Railton (1981, 250-2), and I argue that Railton's nonprobabilistic account has the same flaws as an egalitarian probabilistic account of SM explanation. I then consider the possibility that SM is not explanatory at all.

A terminological note: in the quotation below, Railton talks about statistical mechanics' explanation of the "prevalence of equilibrium". By this he means the almost universal tendency, asserted by the second law of thermodynamics, for a system's entropy to increase until it reaches the maximum possible amount. A gas's spreading throughout a box is an example of this phenomenon; an entirely even distribution is the maximum entropy, or equilibrium, state.

Railton's analysis of explanation in statistical mechanics is in accordance with the pure causal account, described in section 1.3. The role played by high probabilities is now played by non-probabilistic assertions about initial conditions. For expository purposes, I will present the account in two stages. I first describe what I call the brute fact account of sm explanation, and I then show how a suggestion of Railton's augments the brute fact account so as to deflect an obvious objection. The end result is Railton's own view of sM explanation.

The flavor of the brute fact account can be found in the following passage: The prevalence of equilibrium ... must... ultimately be attributed to brute fact and to the operation of deterministic laws on brute fact. But given a certain range of initial conditions, classical statistical mechanics does provide explanations... (Railton 1981. 252).

The brute fact view, then, expressed as a view about the gas in the box, is that the even distribution of the gas is explained by:

1. The brute fact (for which there is no sM explanation) that all gases start out with certain kinds of initial conditions. Call the set of such conditions $C$. 
2. The fundamental laws of physics, according to which any gas starting out with initial conditions in $C$ becomes evenly distributed throughout the box.

In short, the prevalence of equilibrium rather than of non-equilibrium is explained by the brute fact that most gases start out with the kind of initial conditions that, given the laws of physics, lead them to equilibrium.

The brute fact account is open to a familiar kind of objection: if the account is correct, sM would explain flight from equilibrium just as well as approach to equilibrium. Suppose, for example, that my gas huddles in a corner of its box rather than rushing to fill the newly available space when the partition is removed. This unbalanced behavior can be explained by:

1. The brute fact (for which there is no sm explanation) that the gas started out with a certain kind of initial conditions. Call the set of such conditions $\widehat{C}$.

2. The fundamental laws of physics, according to which any gas starting out with initial conditions in $\widehat{C}$ gathers in one corner of the box.

The explanation is just as good-because exactly the same-as the explanation of equilibrium. If this is all there is to sm's explanation of the second law, then the explanation is empty in the sense defined in section 2.3 it provides no understanding of why gases move to equilibrium states rather than non-equilibrium states, hence no understanding of why gases obey the second law of thermodynamics. The historical argument can also be brought to bear: on the brute fact account, Maxwell and Boltzmann added nothing explanatory to the seventeenth century molecular theory.

Railton proposes the following enhancement of the brute fact view. He claims that SM is especially well-suited to explain movement towards equilibrium because

(IC) The fundamental laws of physics and the definition of equilibrium are such that almost all possible sets of initial conditions fall into class 
$C$, the class leading to equilibrium, and almost none into $\widehat{C}$, the class leading to non-equilibrium.

What weight does this observation add to the brute fact explanation of equilibrium?

What Railton says on this subject is not easy to interpret. His view is contained, if anywhere, in the following passage:

This [IC] illuminates a modal feature of the causal processes involved and therefore a modal feature of the relevant ideal explanatory texts: this sort of causal process is such that its macroscopic outcomes are remarkably insensitive ... to wide variations in initial microstates. The stability of an outcome of a causal process in spite of significant variation in initial conditions can be informative about an ideal causal explanatory text in the same way that it is informative to learn, regarding a given causal explanation of the First World War, that a world war would have come about (according to this explanation) even if no bomb had exploded in Sarajevo. This sort of robustness or resilience of a process is important to grasp in coming to know explanations based upon it. (Railton 1981, 251)

(An ideal causal explanatory text for an event is a list of all the laws and particular facts that played a role in bringing about the event, that is, it is a complete pure causal explanation.)

I fully agree with Railton that IC, and more generally "robustness", are central to statistical mechanical explanation. ${ }^{18}$ I will argue, however, that it is impossible to make sense of the explanatory role of robustness without regarding SM explanation as probabilistic, and probabilistic in at least a mildly elitist way.

18. Note that there is more than one notion of "robustness" or "invariance" to be found in the literature on causation and explanation. The notion under discussion here is perhaps closest to that presented in Woodward (1997). 
Nowhere does Railton explain why, if the pure causal account is correct, robustness is of explanatory worth. On the pure causal view, what explains an event are the initial conditions and fundamental laws that brought about the event. The "modal feature" of the causal story, however, concerns other ways that the event might have been caused but was not-the First World War example makes this very clear. What Railton calls a modal property of the causal process, then, would have been better called a counterfactual property. Now why, on a purely causal account of explanation, is information about how an event was not caused explanatorily relevant? This I do not understand.

It may be, however, that my interpretation of Railton as offering a pure causal account of explanation is too narrow. And in any case, the issue here is not whether the pure causal theorist can offer a non-probabilistic account of sM explanation, but whether anyone can. So is there some view of explanation that makes robustness, in Railton's sense, an explanatorily desirable property?

A elitist theory of probabilistic explanation accounts for the explanatory power of robustness very well. In the case of statistical mechanics, because $C$ is much larger than $\widehat{C}$, the initial conditions of a given gas are far more likely to fall into $C$ than $\widehat{C}$. And because initial conditions that lead to equilibrium are far more likely than those that do not, movement towards equilibrium is far better explained than movement away from equilibrium (or on a very strong elitism, such as Hempel's, where low probability events are not explained at all, movement towards equilibrium is explained whereas movement away is not). More generally, robust processes are more likely to be initiated than non-robust processes, thus their outcomes are better explained when they happen. We understand the outbreak of the First World War, for example, when we see that, however things turned out, war was very likely.

If an egalitarian is to resist this view of the explanatory worth of robustness, they must maintain that (using the sm case as an example) what matters is not the high probability of a gas's initial conditions falling into $C$, but the 
great size of $C$. They must then find some account of explanation on which the size, but not the probability, is what makes the explanatory difference.

I think I can show that no such account can succeed, by considering a case in which size and probability pull in opposing directions. Imagine a world in which, in spite of the great size of $C$, the probability of a gas's initial conditions falling into $C$ was actually very small, and that of the conditions falling into $\widehat{C}$ very large. In that world, I propose, it would be movement towards non-equilibrium that would be explicable, and movement towards equilibrium that would be inexplicable, or at least, vastly less explicable. We would understand a prevalence of non-equilibrium, but a prevalence of equilibrium would puzzle us: how could equilibrium be so widespread when the initial conditions leading to equilibrium are so unlikely? No fact about the mere size of the set of initial conditions leading to equilibrium would assuage this worry. I conclude from this example that our explanatory practices respond to the probability, not the size, of sets of initial conditions, and that the higher the probability, the better the explanation. ${ }^{19}$ Robustness matters in explanation because of the relation between the robustness of a process and the high probability of the events brought about by the process. Only an elitist account of explanation can respect this fact. Hence only an elitist account of explanation can make sense of the explanatory importance of IC in statistical mechanics.

The egalitarian might at this point launch the most desperate of defenses and claim that statistical mechanics, because it traffics in fictional probabilities, simply has no explanatory power. It has plenty of predictive power, of course; the claim would be that SM does not explain any of the phenomena

19. It is worth noting that the world I describe is not so imaginary. There are many situations in the real world where a system is overwhelmingly likely to find itself in a very small region of the allowed state space. This happens when both the temperature of the system and the energy of the small region are relatively low, as when a gas condenses or a metal undergoes spontaneous magnetization. It is for this reason that the entropy of a state is defined according to its probability, not its state space volume. 
it predicts. This is certainly a logical possibility, but if true it would have devastating consequences for human understanding.

Suppose I throw a brick at a window, breaking it. Because the fundamental physics of the event is reversible ${ }^{20}$ it is in principle possible for the energy dissipated in the brick's impact to reconverge on the brick and the scattered shards of glass in such a way that they would leap back into the window frame. The brick would fly into my hand and the shards would reconstitute themselves, forming an unbroken pane of glass. Why does this not happen? Because the probability of energy flowing in this way is vanishingly low. But if SM has no explanatory power, this is an empty explanation. It follows that we have no understanding of why windows do not spontaneously reconstitute themselves.

Or consider a fatal car accident. This process, too, is reversible: it is physically possible for the damage to be undone, for the dead to be brought back to life. The explanation of why this does not happen is an sm explanation. If sm does not explain, we have no understanding of why accident victims are not resurrected every day.

The void extends to more theoretical cases. Our understanding of life depends on our understanding of biochemical reactions. But, like all chemical reactions, these are explained in part by thermodynamics, hence in part by statistical mechanics. If sM conveys no understanding, then neither does biochemistry, in which case we have no understanding of the processes underlying respiration, nutrition, reproduction, and so on. I would say that an extremely high proportion-perhaps over $90 \%$, although I am only guessing - of scientific work published today depends for its explanatory power at least implicitly on some principle grounded in SM. The egalitarian who denies that SM explains may well be proposing to cut our understanding

20. There is an interpretation of quantum mechanics on which the physics is not reversible, but on this interpretation, the probabilities of statistical mechanics are quantum mechanical probabilities (Albert 1994). This would undercut any of the usual arguments that the probabilities of sm are not real. Proponents of irreality must accept reversibility. 
of the world by a factor of ten. Is an aging philosophical doctrine about probabilistic explanation worth this sacrifice? Surely it is better to look for something philosophically new.

\section{What Went Wrong?}

Why has egalitarianism gained so much currency, given its inadequacies? One reason is that the pure causal account has become widely accepted, and as I showed in section 1.3, egalitarianism is difficult for a pure causal theorist to avoid. But this raises a new question: why has the pure causal account gained so much currency, given the inadequacies of egalitarianism? The answer, at least in part, is that egalitarianism itself has considerable appeal, a phenomenon for which I give two explanations.

The first reason for the egalitarian consensus is quite complex. It issues from the fact that probabilistic processes are simultaneously anarchic and lawful: they are quite chaotic in the short term, almost entirely unpredictable from one moment to the next, but very stable in the long term, producing (almost always) frequencies of outcomes roughly equal to the relevant probability. ${ }^{21}$ You cannot predict whether the next toss of a fair coin will land heads or tails, but you can predict with great confidence that over the course of a thousand tosses, about half will land heads. A probabilistic explanation, then, can explain either short term disorder or long term order.

The high probability explanations that I have discussed in this paper, those of statistical mechanics, are of the second sort: they derive their power from the capacity of probabilistic processes to produce certain kinds of order in nature. Indeed, the second law of thermodynamics is just a domain-specific version of the principle that, in the long run, frequencies tend to match

21. Probabilistic processes involving more than one probability, such as Markov chains, have their own, slightly more complicated, long term stability properties. 
probabilities..$^{22}$ I assert more generally that all high probability explanations appeal to the long term stability-inducing capacity of probability.

Egalitarianism has been able to gain a hold, I suggest, because philosophers working on probabilistic explanation have almost entirely ignored the explanatory power that probabilistic processes possess in virtue of their capacity to induce order. Philosophers have thus ignored the explanatory power of high probability.

The reason for this is, I suspect, in great part an unrepresentative choice of examples. Typically, work on probabilistic explanation focuses on the probabilities found in quantum mechanics, in medical journals, and in statistical social science. Theories in these domains are probabilistic largely because we do not understand the lower level mechanisms at work (in the case of quantum mechanics, perhaps because there are no lower level mechanisms at work). In models of these domains, probability stands in for ignorance: the short term disorder of probabilistic processes captures the apparent disorder of a complex system that we do not understand. It is not clear whether a probability playing such a role is explanatory at all, but even if it is, this sort of example illustrates only the capacity of probability to explain disorder.

By far the more interesting use for probability is the explanation of order. That complicated systems behave in ways that are difficult to predict in the short term is not very surprising; providing a mathematical surrogate for this unpredictability hardly counts as a great explanatory feat. That complicated systems behave in orderly ways in the long term, on the other hand, is a strange and wonderful thing. Where probability helps to account for this order, as in statistical mechanics, we have some of the most compelling explanations in all of science. Yet one would be hard put to find any substantial reference to statistical mechanics in the influential papers of Hempel, Salmon, or Humphreys.

This blindness to the deep explanatory powers of probability has caused,

22. This is true, at least, on probabilistic or information-theoretic definitions of entropy. 
in the minds of egalitarians, the atrophy of the notion of probability itself. Because they consider only cases of explanation where probability is employed to plug a gap in our understanding, egalitarians have come to equate probabilistic processes with processes that are not governed by any nomological principle at all. (For more on the distinction between probabilistic processes and processes entirely unconstrained by law-the distinction elided by the egalitarians-see Earman (1986).) As a result, egalitarians end up with a notion of probability so slender that it is no wonder they do not wish it to bear any explanatory weight. There is much evidence for this conceptual wasting in the literature. Consider, for example, the following observations, crucial to the authors' arguments for egalitarianism:

... where a statistical explanation is appropriate, there's no reason for the [occurrence of the explanandum]: it came about by chance (Jeffrey 1969, 24).

If there were a reason why one probabilistic outcome of a chance process was realized rather than another, we would not be dealing with a chance process (Railton 1981, 238).

What ... do we have to replace the traditional account of explanatory knowledge?... When we know of all the causes, then we shall know all there is to know of the kind with which we are concerned, for all that will be left is pure chance, and chance is, as I have said, literally nothing (Humphreys 1989, 141).

In Humphreys' work, at least, the concept of probability has entirely withered away.

The way "chance" is used in these passages is the way it is used by, for example, Lucretius in his exposition of the Epicurean doctrine of the clinamen or "lucky swerve". What happens by chance happens not within the bounds of law but in the gaps left ungoverned by law, by nature's whim. ${ }^{23}$ The

23. The laws may determine which outcomes are possible, but they say nothing about 
reader will recall that the Epicureans used the clinamen to explain, first, chaos (the atoms collide rather than raining endlessly downward), and second, order (the structure of objects, including organisms, is a chance product of colliding atoms). Of these it is well suited to account for chaos, because it is in essence lawlessness. It yields a poor explanation of order, howevernot only because it cannot account for complex structures, but because it cannot account for any kind of structural stability at all. Yet one of the most fruitful scientific uses of physical probability, I have argued-statistical mechanics - is to explain stability, in the form of regularities such as the second law of thermodynamics and the ideal gas laws. Physical probability has this explanatory power because, unlike the clinamen, it is in essence lawful: behavior described by physical probabilities is governed by statistical law.

To equate physical probability with the nomological vacuum of the clinamen, then, is to relinquish the power of probability to explain stability, and thereby to ignore that facet of a probability ascription that quantifies the stability property- the value of the probability. It is not surprising, then, that the egalitarians, treating probabilistic processes as if they were governed by no principle whatsoever, conclude that probability values are unimportant. Nor is it surprising that egalitarian explanation is utterly unable to account for the kinds of order in our universe that are brought about by probabilistic processes- such as the patterns that are described by the second law of thermodynamics.

Wesley Salmon writes that the idea that high probabilities explain better than low probabilities is an "anachronistic carryover" from Laplacean determinism (Salmon 1984, 113). Like his fellow egalitarians, he misses the beauty of probabilistic explanation. What is so fascinating about probability is that it lies neither in the domain of Laplacean determinism nor in that of Epicurean anarchy, but somewhere in between, where short term disorder

which of the possible outcomes will occur. 
and long term order meet.

The second reason for the egalitarian consensus is a tacit assumption that there is just one kind of probabilistic explanation. Writers look at events with low probability and exclaim that they are surely not inexplicable. If they are explicable, then there must be a kind of explanation according to which even events of low probability are explained. But this kind of explanation obviously does not care too much about the values of probabilities. Now, add the assumption that there is only one kind of probabilistic explanation, and it follows that the one and only kind of probabilistic explanation does not care about probability values, and so is egalitarian. A good example of this enthymematic line of reasoning can be found in Railton:

Jeffrey is surely right, as against Hempel, that probable and improbable outcomes of deterministic processes are equally explicable, and explicable in the same way. After all, why should it be explicable that a genuinely random wheel of fortune with 99 red stops and 1 black stop came to a halt on red, but inexplicable that it halted on black? (Railton 1978, 212)

The rhetorical question that serves as the premise ("After all...") is only relevant to the conclusion ("that probable and improbable events...") if it is assumed that a single paradigm must make sense of every probabilistic explanation. ${ }^{24}$

I will conclude by advocating a moderate elitism in probabilistic explanation. The paresis case shows that high probabilities are not necessary for explanation. But the nature of probabilistic explanation in statistical mechanics shows that high probabilities explain better than low probabilities. The correct account of explanation will underwrite both of these conclusions. Possibly, although not necessarily, there will turn out to be two or

24. For a different view on how to have explanation of low probability events without egalitarianism, see Sober (1984 143-5). 
more forms of probabilistic explanation. One form-perhaps the pure causal theory itself-will account for the explanatory power of low probabilities; another will account for the superior explanatory power of high probabilities. In the place of egalitarianism, then-pluralism. 


\section{References}

Albert, D. Z. (1994). The foundations of quantum mechanics and the approach to thermodynamic equilibrium. British Journal for the Philosophy of Science 45:669-677.

Brush, S. G. (1983). Statistical Physics and the Atomic Theory of Matter from Boyle and Newton to Landau and Onsager. Princeton University Press, Princeton, NJ.

Earman, J. (1986). A Primer on Determinism. D. Reidel, Dordrecht.

van Fraassen, B. C. (1980). The Scientific Image. Oxford University Press, Oxford.

Hempel, C. G. (1965a). Aspects of scientific explanation. In Hempel (1965b), chap. 12, pp. 331-496.

—. (1965b). Aspects of Scientific Explanation. Free Press, New York.

Hempel, C. G. and P. Oppenheim. (1948). Studies in the logic of explanation. Philosophy of Science 15:135-175. Reprinted in Hempel (1965b), chap. 10.

Humphreys, P. (1981). Aleatory explanations. Synthese 48:225-232.

—. (1989). The Chances of Explanation. Princeton University Press, Princeton, NJ.

Jeffrey, R. C. (1969). Statistical explanation vs. statistical inference. In N. Rescher (ed.), Essays in Honor of Carl G. Hempel, pp. 104-113. D. Reidel, Dordrecht. Reprinted in Salmon et al. (1971).

Mellor, D. H. (1976). Probable explanation. Australasian Journal of Philosophy 54:231-241.

Papineau, D. (1985). Probabilities and causes. Journal of Philosophy 82:57-74. 
Railton, P. (1978). A deductive-nomological model of probabilistic explanation. Philosophy of Science 45:206-226.

—. (1981). Probability, explanation, and information. Synthese 48:233256.

Salmon, W. C. (1970). Statistical explanation. In R. G. Colodny (ed.), The Nature and Function of Scientific Theories, pp. 173-231. University of Pittsburgh Press, Pittsburgh. Reprinted in Salmon et al. (1971).

- (1984). Explanation and the Causal Structure of the World. Princeton University Press, Princeton, NJ.

- (1988). Deductivism visited and revisited. In A. Grünbaum and W. C. Salmon (eds.), The Limits of Deductivism, pp. 95-127. University of California Press, Berkeley, CA.

—_. (1990). Four Decades of Scientific Explanation. University of Minnesota Press, Minneapolis.

Salmon, W. C., R. Jeffrey, and J. Greeno. (1971). Statistical Explanation and Statistical Relevance. University of Pittsburgh Press, Pittsburgh.

Scriven, M. (1959). Explanation and prediction in evolutionary theory. Science 30:477-482.

Sklar, L. (1993). Physics and Chance. Cambridge University Press, Cambridge. Sober, E. (1984). The Nature of Selection. MIT Press, Cambridge, MA.

Woodward, J. (1997). Explanation, invariance, and intervention. Philosophy of Science 64:S26-S41. 